\title{
Simulation of open-quantum-system dynamics using the quantum Zeno effect
}

\author{
Sabrina Patsch $\odot,{ }^{1,2}$ Sabrina Maniscalco, ${ }^{3,4}$ and Christiane P. Koch $\oplus^{1,2, *}$ \\ ${ }^{1}$ Theoretische Physik, Universität Kassel, Heinrich-Plett-Straße 40, D-34132 Kassel, Germany \\ ${ }^{2}$ Dahlem Center for Complex Quantum Systems and Fachbereich Physik, Freie Universität Berlin, Arnimallee 14, D-14195 Berlin, Germany \\ ${ }^{3}$ QTF Centre of Excellence, Turku Centre for Quantum Physics, Department of Physics and Astronomy, \\ University of Turku, FIN-20014 Turku, Finland \\ ${ }^{4}$ QTF Centre of Excellence, Department of Applied Physics, Aalto University, FIN-00076 Aalto, Finland
}

(Received 8 July 2019; accepted 8 April 2020; published 6 May 2020)

\begin{abstract}
We suggest a quantum simulator that allows to study the role of memory effects in the dynamics of open quantum systems. A particular feature of our simulator is the ability to engineer both Markovian and non-Markovian dynamics by means of quantum measurements and the quantum Zeno dynamics induced by them. The simulator is realized by two subsystems of a bipartite quantum system or two subspaces of a single system which can be identified as system and meter. Exploiting the analogy between dissipation and quantum measurements, the interaction between system and meter gives rise to quantum Zeno dynamics, and the dissipation strength experienced by the system can be tuned by changing the parameters of the measurement, i.e., the interaction with the meter. Our proposal can readily be realized with existing experimental technology, such as cavity- or circuit-QED platforms or ultracold atoms.
\end{abstract}

DOI: 10.1103/PhysRevResearch.2.023133

\section{INTRODUCTION}

Quantum simulation uses a controllable quantum system to study another less controllable quantum system [1]. It promises to advance the study of many-body dynamics in condensed matter physics [1], but application to open quantum systems is also most natural [2-19]. Realizations to date have focused on qubit dynamics, encoding the system in trapped ions [3], photon polarization $[4,6,16,18]$, superconducting qubits [19], or nuclear spins [10], but more complex systems such as the light harvesting complex have also been suggested [5]. A key interest in the quantum simulation of open quantum systems is a controllable transition from Markovian to nonMarkovian dynamics, as quantified by one of the various non-Markovianity measures [20-22]. This is motivated in a twofold way. First, non-Markovian dynamics are ubiquitous in condensed phase [23], encountered in settings as different as light harvesting or solid-state based quantum technologies, but inherently difficult to describe and study [24]. Second, memory effects may present an exploitable resource [25-29], allowing, for example, for more efficient cooling [30], better quantum communication [31,32], and improved quantum gate operation [33,34]. For memory effects to appear in a multi-partite open system quantum simulator, one needs to engineer suitable environmental correlations [10] or a sufficiently strong interaction of system and environment. The

\footnotetext{
*christiane.koch@fu-berlin.de

Published by the American Physical Society under the terms of the Creative Commons Attribution 4.0 International license. Further distribution of this work must maintain attribution to the author(s) and the published article's title, journal citation, and DOI.
}

latter can be achieved with superconducting qubits encoding both system and environment $[5,19]$. What is missing to date is an open system quantum simulator that allows us to fully tune the strength of memory effects. This is essential to study the role of non-Markovianity in the engineering and control of open quantum systems.

Here, we fill this gap by providing a framework for inducing and tailoring open system dynamics with tunable non-Markovianity and dissipation. Our protocol is based on quantum measurements and exploiting quantum Zeno dynamics, by coupling a system to a meter which is measured destructively after a short interaction time, cf. Fig. 1(a). In the limit of quasi-continuous measurements, the system dynamics is confined to a specific subspace, i.e., it turns into quantum Zeno dynamics [35]. We show here that the control available in the measurement process can be used to simulate the effect of an engineered environment, with full tunability of the system dynamics from being memoryless to exhibiting effects of memory. Moreover, in the Markovian limit, we can generate arbitrary open system dynamics.

\section{THEORETICAL FRAMEWORK}

For simplicity, we take the system to be a harmonic oscillator, $\hat{H}_{\mathrm{s}}=\sum_{n} \omega n|n\rangle\langle n|$, where we have set the vacuum energy to zero ( $\hbar=1$ throughout). Note that considering a finite $n$-level system instead leads to qualitatively the same results (cf. Appendix B). The system is coupled to a meter which we model by a three-level system with states $|h\rangle,|g\rangle$, and $|e\rangle, \hat{H}_{\mathrm{m}}=\omega^{\prime}|g\rangle\left\langle g\left|+\left(\omega+\omega^{\prime}\right)\right| e\right\rangle\langle e|$. The energy of $|h\rangle$ is set to zero and the $|g\rangle \leftrightarrow|e\rangle$ transition is resonant with the system frequency $\omega$ while the $|h\rangle \leftrightarrow|g\rangle$ transition is far off-resonant with $\omega \ll \omega^{\prime}$. The meter can easily consist of more than three levels but existence of one resonant and at 

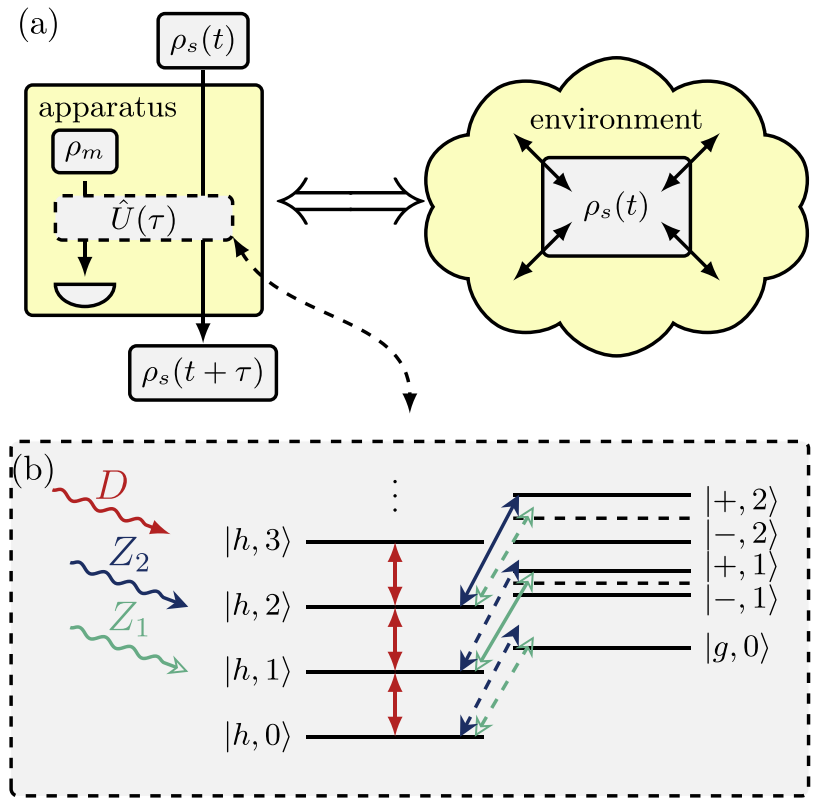

FIG. 1. (a) Analogy between indirect measurement of a system by a meter (left) and an open system (right). (b) Energy level diagram of a bipartite system consisting of a harmonic oscillator and a fewlevel system, interacting with three external fields-a drive $D$ of the harmonic oscillator (red) and two Zeno pulses $Z_{1}$ (green, open notched arrowhead) and $Z_{2}$ (blue, notched arrowhead) addressing the levels $|z=1\rangle$ and $|z=2\rangle$. Arrows with solid (dashed) lines indicate (off-)resonant transitions.

least one off-resonant transition is essential. The resulting coupling, in the interaction picture with respect to the drift Hamiltonian, $\hat{H}_{0}=\hat{H}_{\mathrm{s}}+\hat{H}_{\mathrm{m}}$, and using the rotating wave approximation, can then be described by a Jaynes-Cummings type interaction [36],

$$
\hat{H}_{\mathrm{sm}}=\frac{\Omega}{2}\left(\hat{\sigma}_{-} \hat{a}^{\dagger}+\hat{\sigma}_{+} \hat{a}\right)
$$

with Rabi frequency $\Omega, \hat{\sigma}_{-}=|g\rangle\langle e|=\hat{\sigma}_{+}^{\dagger}$, and creation and annihilation operators $\hat{a}^{(\dagger)}$ for the harmonic oscillator. The eigenstates of $\hat{H}_{\mathrm{sm}}$ are the dressed states $|g, 0\rangle$, and $| \pm, n\rangle=$ $\frac{1}{\sqrt{2}}(|e, n-1\rangle \pm|g, n\rangle)$ for $n \geqslant 1$ with eigenenergies $E_{n}^{ \pm}=$ $\omega n \pm \frac{\Omega}{2} \sqrt{n}$ [36], cf. Fig. 1(b). In addition to its coupling to the meter, the system is driven by a resonant classical source $D$ with field strength $\alpha$,

$$
\hat{H}_{\mathrm{D}}=\alpha \hat{a}^{\dagger}+\alpha^{*} \hat{a} .
$$

We assume the drive to be weak compared to the coupling with the meter, $\alpha \ll \Omega$, such that the system will only be driven if the meter is in $|h\rangle$. In this limit, $\hat{H}_{\mathrm{D}}$ will induce a displacement $\beta=-i \alpha \tau$ of the system state, $\hat{U}_{\mathrm{D}}(\tau)=e^{-i \hat{H}_{\mathrm{D}} \tau}=$ $e^{\beta \hat{a}^{\dagger}-\beta^{*} \hat{a}}$.

A tunable source of dissipation is introduced by a series of indirect measurements of the system state using identical meters, cf. Fig. 1(a). These measurements will determine whether the system is in a specific Fock state $|z\rangle$, the "Zeno level." To this end, system and meter are coupled by a so-called Zeno pulse $Z_{z}$ with coupling strength $g_{z}$ which is resonant to the $|h, z\rangle \leftrightarrow|+, z\rangle$ transition,

$$
\hat{H}_{z}=\frac{g_{z}}{2}(|h, z\rangle\langle+, z|+|+, z\rangle\langle h, z|),
$$

cf. Fig. 1(b). $Z_{z}$ will induce Rabi oscillations only if the system is in the Zeno level, $\hat{U}_{\mathrm{z}}(\tau)=e^{-i \hat{H}_{z} \tau}=e^{(|h, z\rangle\langle+, z|+|+, z\rangle\langle h, z|) \phi_{z} / 2}$, accumulating a Rabi angle $\phi_{z}=g_{z} \tau$ during the interaction time $\tau$. Thus there will only be population in the state $|+, z\rangle$ if there was population in $|z\rangle$ initially. A subsequent destructive measurement of the meter at time $\tau$, corresponding to a partial trace over the meter, thus provides information on the population of $|z\rangle$ [35]. Due to the anharmonicity of the dressed states it is straightforward to employ several Zeno pulses $Z_{z_{i}}$ addressing multiple levels $\left|z_{i}\right\rangle$ at the same time, with Hamiltonian $\hat{H}_{Z}=\sum_{i=1}^{N_{Z}} \hat{H}_{z_{i}}$ where $N_{Z}$ is the number of Zeno pulses employed. The system state at the end of one time interval $\tau$ can be obtained in terms of the piecewise dynamics,

$$
\hat{\rho}_{\mathrm{s}}(t+\tau)=\operatorname{Tr}_{\mathrm{m}}\left\{\hat{U}(\tau) \hat{\rho}(t) \hat{U}^{\dagger}(\tau)\right\}
$$

with $\hat{U}(\tau)=e^{-i\left(\hat{H}_{\mathrm{D}}+\hat{H}_{\mathrm{sm}}+\hat{H}_{z}\right) \tau}$ and $\hat{\rho}\left(\hat{\rho}_{\mathrm{s}}\right)$ describing the bipartite (reduced) state. The sequence of coupling the system to a meter during time $\tau$ and performing a destructive measurement of the meter afterwards is repeated several times, with the new meter initially always in $|h\rangle$. For small displacements of the system between two measurements, $|\beta| \ll 1$, the protocol gives rise to quantum Zeno dynamics (QZD) [35,37,38]. Hence, when choosing an initial state of the system below the Zeno level, $\left|n_{0}<z\right\rangle$, the dynamics is confined to the Zeno subspace $\mathcal{H}_{z}=\{|0\rangle, \ldots,|z-1\rangle\}$. The displacement $\beta$ (due to the drive $D$ ) and the Rabi angle $\phi_{z}$ (due to the Zeno pulse $Z_{z}$ ) act as "knobs" to control the dynamics of the quantum simulator as we show below.

\section{IMPLEMENTATION OF THE OPEN-QUANTUM-SYSTEM SIMULATOR}

To illustrate the implementation of the simulator, we choose the overall protocol duration $T$ such that $-i \alpha T=$ $2 \pi$ and we take the initial state to be $\left|n_{0}=0\right\rangle$. To quantify the amount of dissipation, we use the linear entropy, $S_{L}=$ $1-\operatorname{Tr}\left\{\hat{\rho}_{\mathrm{s}}^{2}\right\}$. For the following discussion and explanation of the system dynamics, it also proved to be useful to define a measure for the infidelity of the QZD. To this end, we introduce the population $P_{\bar{Z}}$ that has escaped from the Zeno subspace, $P_{\bar{Z}}=\sum_{n=z}^{\infty}\left\langle n\left|\hat{\rho}_{\mathrm{s}}(T)\right| n\right\rangle$. In addition to that, we expect the system dynamics to display memory effects and use the so-called BLP measure quantifying non-Markovianity as accumulated revivals in distinguishability of two initial states [39]. The optimal state pair which maximizes the BLP measure for our choice of $\alpha$ and $|z\rangle$ is given by

$$
\left|\Psi_{1}(\vartheta)\right\rangle=\cos \left(\frac{\vartheta}{2}\right)|0\rangle+\sin \left(\frac{\vartheta}{2}\right)|1\rangle, \quad\left|\Psi_{2}\right\rangle=|2\rangle
$$

for all $\vartheta \in[0,2 \pi)$ as can be shown by analytical reasoning in Appendix A, and we take $\vartheta=0$ in the following.

\section{A. Implementation with one Zeno pulse}

We start by discussing the quantum simulator subject to the drive $D$ (which controls the displacement $\beta$ ) and one 


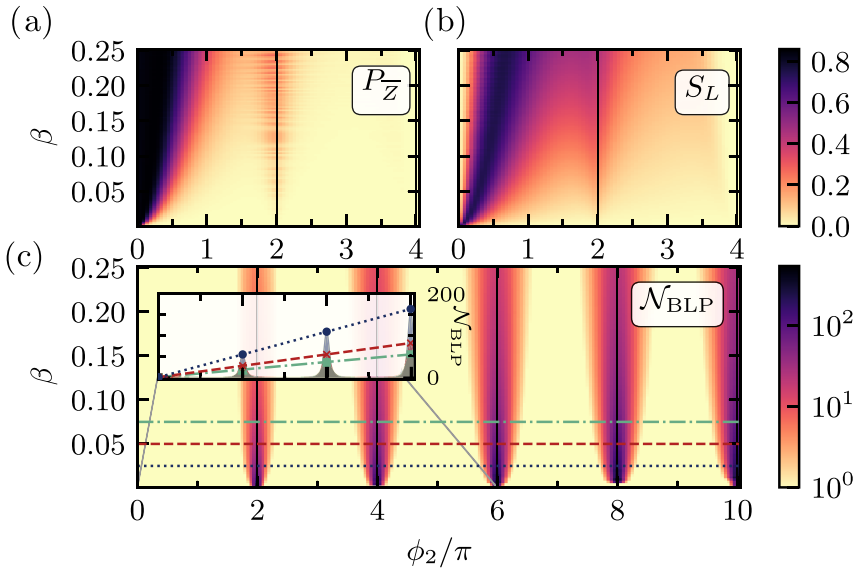

FIG. 2. Infidelity $P_{\bar{Z}}$ of the quantum Zeno dynamics (a), dissipation $S_{L}$ (b), and non-Markovianity measure $\mathcal{N}_{\mathrm{BLP}}$ (c) as a function of the displacement $\beta$ due to the drive $D$ and the Rabi angle $\phi_{2}$ accumulated due to the Zeno pulse $Z_{2}$. (Inset) $\mathcal{N}_{\mathrm{BLP}}$ as a function of $\phi_{2}$ for $\beta=0.025$ (blue dotted, circles, highest peaks), 0.050 (red dashed, crosses), and 0.075 (green dash-dotted, squares, smallest peaks) with linearly increasing peak heights. The data points which generate the linear functions were calculated analytically.

Zeno pulse $Z_{z=2}$ addressing the Zeno level $|z=2\rangle$ (which controls the Rabi angle $\phi_{z=2}$ ), cf. Fig. 2. An arbitrary degree of dissipation, indicated by $S_{L}$, can be engineered by tuning $\beta$ and $\phi_{2}$. The figure shows that there is a clear correlation between the dissipation and the infidelity of the QZD, $P_{\bar{Z}}$. In particular, strong dissipation can be realized by a small $\phi_{2}$ where the population leaves the Zeno subspace. Only in this case, the Zeno level $|2\rangle$, which is the only state being subject to dissipation due to the Zeno coupling, is populated. Hence, it is constructive to understand the mechanisms leading to Zeno confinement in more detail as there is more than one.

(i) QZD describes the effect of confining the dynamics of a system to a tailored subspace by frequent measurements. The dynamics shown in Fig. 2 agrees with this intuitive picture since the Zeno infidelity $P_{\bar{Z}}$ vanishes as the displacement $\beta$ (which is proportional to the time between two measurements) goes to zero.

(ii) QZD can not only be induced by frequent measurements but also by strongly coupling the system to a meter. In fact, the two cases are formally equivalent in the limit of a fast repetition rate and strong coupling [40]. In our case, the coupling strength between the harmonic oscillator and the three-level system is given by the field strength of the Zeno pulse, $g_{z}=\phi_{z} / \tau$, which is proportional to $\phi_{z}$. Thus, the quality of the Zeno confinement is very good for large values of the Rabi angle $\phi_{z}$ even if $\beta$ is large. For Rabi angles $\phi_{z}>4 \pi, P_{\bar{Z}}$ and $S_{L}$ are vanishingly small which is why this parameter range is omitted in Figs. 2(a) and 2(b).

(iii) The coupling between the Zeno level and the dressed state $|+, z\rangle$ induces a decay of the Zeno level population to the level below, that is, back into the Zeno subspace. More explicitly, the Hamiltonian $\hat{H}_{z}$ [cf. Eq. (3)] will induce Rabi oscillations

$$
\left|\Psi_{|h, z\rangle}(t)\right\rangle=\cos \left(\frac{g_{z} t}{2}\right)|h, z\rangle+\sin \left(\frac{g_{z} t}{2}\right)|+, z\rangle,
$$

while all other states $|h, n \neq z\rangle$ are unaffected by $\hat{H}_{z}$. The action of the drive $D$ is negligible on the timescale $\tau$ since $\alpha \ll g_{z}$. When performing a Zeno pulse with Rabi angle $\phi_{z}=$ $g_{z} \tau \neq 2 n \pi$, a fraction of $\sin ^{2}\left(\frac{\phi_{z}}{2}\right)$ of the population that has been in the state $|h, z\rangle$ ends up in the state $|+, z\rangle$. According to the definition of the dressed states, half of the system is in the state $|z-1\rangle$ here, i.e., back in the Zeno subspace, which enhances the Zeno confinement. As a consequence, if the initial state is lying above the Zeno level, $n_{0}>z$, the Zeno pulse has to be changed to be resonant to the $|h, z\rangle \leftrightarrow$ $|+, z+1\rangle$ transition, to also benefit from this enhancement.

(iv) For $\pi<\phi_{z}<2 \pi$, the phase of the $|h, z\rangle$ contribution in Eq. (6) is negative due to the cosine. This leads to an inversion of the dynamics back into the Zeno subspace which improves the QZD additionally.

Note that there is one exception to the correlation between dissipation $S_{L}$ and the Zeno infidelity $P_{\bar{Z}}$. For $\phi_{2} \rightarrow 0$, there is no dissipation even though the population leaves the Zeno subspace. In this case, the coupling between system and meter vanishes and the system evolves freely without dissipation.

The signatures of non-Markovianity are strongest for Rabi angles $\phi_{2}=2 n \pi$ with $n$ being an integer, cf. Fig. 2(c). This is because for small $\beta$ and every choice of $|z\rangle$, a pulse $Z_{z}$ with $\phi_{z}=2 n \pi$ corresponds to the map $|h, z\rangle \mapsto(-1)^{n}|h, z\rangle$, i.e., the pulse changes only the phase of the Zeno level and no entanglement between system and meter is generated. In other words, this choice of $\phi_{z}$ results in a quantum nondemolition measurement [41] preserving the information of the system's state. In this case, the value of the non-Markovianity measure can be calculated analytically (cf. Appendix A). The agreement between the analytical and the numerical calculation is remarkable, as can be seen by the data points in the inset of Fig. 2. They reveal a linear increase of the non-Markovianity measure with Rabi angle $\phi_{2}$ for a given displacement $\beta$. For constant $\phi_{2}$, in turn, the non-Markovianity measure decreases as $\beta$ grows larger because the number of measurements $N=$ $T / \tau \propto T / \beta$, and hence the number of $2 n \pi$-pulses for fixed $T$ decreases. If $N$ is fixed instead of $T$, the value of the nonMarkovian measure is roughly constant for a fixed value of $\phi_{2}$.

\section{B. Realizing the Markovian limit}

For arbitrary values of $z$ and Rabi angles $\phi_{z} \neq 2 n \pi$, entanglement remains between system and meter at the end of each time interval $\tau$. Measurement of the meter then erases information, resulting in less distinguishable states, such that the system dynamics becomes Markovian. As shown in more detail in Appendix B, the dynamics of the harmonic oscillator in the Markovian limit can be described by a master equation by assuming quasi-continuous measurements,

$$
\begin{aligned}
\frac{d \hat{\rho}_{\mathrm{s}}(t)}{d t}= & -i\left[\hat{H}_{\mathrm{D}}, \hat{\rho}_{\mathrm{s}}(t)\right]+\kappa \gamma_{A}\left(\hat{A} \hat{\rho}_{\mathrm{s}}(t) \hat{A}^{\dagger}-\frac{1}{2}\left\{\hat{A}^{\dagger} \hat{A}, \hat{\rho}_{\mathrm{s}}(t)\right\}\right) \\
& +\kappa \gamma_{\Pi}\left(\hat{\Pi} \hat{\rho}_{\mathrm{s}}(t) \hat{\Pi}^{\dagger}-\frac{1}{2}\left\{\hat{\Pi}^{\dagger} \hat{\Pi}, \hat{\rho}_{\mathrm{s}}(t)\right\}\right)
\end{aligned}
$$

with the measurement rate $\kappa=1 / \tau, \quad \hat{\Pi}=|z\rangle\langle z|, \quad \hat{A}=$ $|z-1\rangle\langle z|$, and

$$
\gamma_{A}=\frac{1}{2} \sin ^{2} \frac{\phi_{z}}{2}, \quad \gamma_{\Pi}=4 \sin ^{4} \frac{\phi_{z}}{4}+\gamma_{A} .
$$


Given the definitions of $\hat{A}$ and $\hat{\Pi}, \kappa \gamma_{A}$ corresponds to the rate of population transfer from the Zeno level to the level below, and $\kappa \gamma_{\Pi}$ to the dephasing rate of the Zeno level. The effect of the operator $\hat{A}$ can be identified with the projection the population back into the Zeno subspace as explained in Sec. III A, (iii). This becomes apparent by comparing the decay rate $\gamma_{A}$ with the weight of the $|+, z\rangle$ contribution in Eq. (6).

The master equation provides yet another angle, in addition to Fig. 2, to illustrate the functionality of the quantum simulator: The decay rates can be varied by tuning the experimentally accessible parameters $\phi_{z}$ and $\kappa$. Eq. (7) can be generalized in several ways as shown explicitly in Appendix B: taking the Zeno pulse to couple $|h, z\rangle$ to $|+, n\rangle$ with arbitrary $z$ and $n$, it is possible to generate Lindblad operators $|z\rangle\langle z|| n\rangle,\langle z|$ and $|n-1\rangle\langle z|$ with tunable decay rates. For example, by choosing $n=z+1$, the situation is similar to the one presented above but inducing population excitation, $|z+1\rangle\langle z|$, instead of relaxation, $|z-1\rangle\langle z|$. Choosing the system to be off-resonant with respect to the $|g\rangle \leftrightarrow|e\rangle$ transition of the meter provides more freedom to adjust the decay rates. Taking the Zeno pulse to couple to the $|h, z\rangle \leftrightarrow|e, n\rangle$ transition allows to arbitrarily tune the ratio between dephasing $|z\rangle\langle z|$ and population transfer $|n\rangle\langle z|$. In particular, it allows for inducing pure dephasing in the special case of $n=z$. Finally, employing several pulses at the same time engineers arbitrary decay channels for every system state.

\section{Implementation with two Zeno pulses}

With a single Zeno pulse, dissipation and memory effects cannot be tuned independently. This shortcoming is remedied by employing two state-selective excitations simultaneously, for example $Z_{1}$ and $Z_{2}$ addressing states $|1\rangle$ and $|2\rangle$, cf. Fig. 1 (in principle, any two nondegenerate states can be chosen). Even with two Zeno fields, only a single measurement needs to be carried out at the end of time interval $\tau$. While $Z_{2}$ controls memory effects, $Z_{1}$ induces dissipation: large memory effects are obtained with a Rabi angle $\phi_{2}=2 n \pi$, and small $\phi_{1}$ results in strong dissipation (for our values of the displacement $\left.\beta, \phi_{1}<\pi\right)$. The interplay of two pulses in the non-Markovian regime requires a numerical analysis. As shown in Fig. 3, almost any combination of dissipation strength, quantified by the linear entropy $S_{L}$, and memory effects, quantified by the measure $\mathcal{N}_{\text {BLP }}$ and calculated with $\vartheta=\pi$ (cf. Appendix A), can be realized by varying the displacement $\beta$ and the two Rabi angles $\phi_{2}$ and $\phi_{1}$. The gray dots in Fig. 3 show all combinations of $S_{L}$ and $\mathcal{N}_{\mathrm{BLP}}$ obtained by sampling $\beta$ from 0.01 to 0.5 in 80 steps, $\phi_{2}$ from $2 \pi$ to $12 \pi$ in steps of $2 \pi$ and $\phi_{1}$ from 0 to $2 \pi$ in steps of $0.01 \pi$. For simplicity, we consider the example of $\phi_{2}=4 \pi$ and $\phi_{1} \leqslant \pi$ in more detail (indicated by the colored lines in Fig. 3). For small values of $\phi_{1}$, the dissipation $S_{L}$ is tuned by $\phi_{1}$ while keeping the non-Markovianity almost constant, as can be seen from the initially flat curves. For larger $\phi_{1}$, the curves reach a clear boundary at a linear entropy of $S_{L}=1 / 2$, i.e., at the purity of the completely mixed two-level system state. This is because $Z_{2}$ creates a perfect Zeno barrier at $|2\rangle$ while $Z_{1}$ induces dissipation in the resulting two-dimensional Zeno subspace whose linear entropy is limited by $1 / 2$. When increasing $\phi_{1}$ even further, the pulse $Z_{1}$ induces a Zeno barrier

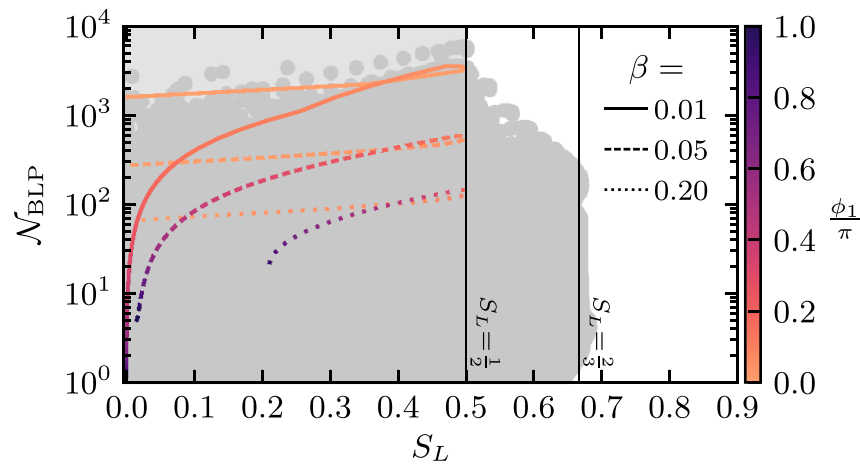

FIG. 3. Attainable combinations of dissipation $S_{L}$ and nonMarkovianity $\mathcal{N}_{\text {BLP }}$ using two Zeno pulses $Z_{2}$ and $Z_{1}$ with $-i \alpha T=$ $10 \pi$ (dark grey dots). The colored lines highlight the combinations of $S_{L}$ and $\mathcal{N}_{\mathrm{BLP}}$ for a Rabi angle $\phi_{2}=4 \pi$ with different values of displacement $\beta$ and Rabi angle $\phi_{1} \leqslant \pi$. The light gray shading highlights the area which is attainable by an extended sampling range.

at $|1\rangle$, and dissipation and non-Markovianity drop drastically. Varying $\phi_{2}$, on the other hand, will tune the non-Markovianity linearly, similar to what is shown in the inset of Fig. 2. Since there is no fundamental limit to the value of $\mathcal{N}_{\mathrm{BLP}}$, the light gray area in Fig. 3 is also accessible by extending the sampling range, in particular the value of $\phi_{2}$.

\section{Tunability of the simulator}

In the case of two combined Zeno pulses, $Z_{1}$ and $Z_{2}$, the dynamics depends on three control parameters $\left(\beta, \phi_{1}, \phi_{2}\right)$ where $\beta$ is the system displacement induced by the coherent drive $D$. Since the values of all three parameters affect both the dissipation strength and the non-Markovianity, the discussion of the tunability of the quantum simulator needs some special attention. In the following, we provide a detailed analysis of the dependency of the linear entropy $S_{L}$, quantifying the dissipation strength, and the non-Markovianity measure $\mathcal{N}_{\text {BLP }}$ on the set of control parameters $\left(\beta, \phi_{1}, \phi_{2}\right)$. Note that the value of the linear entropy $S_{L}$ at a fixed final time $T$ can be interpreted as different strengths in the respective decay rates.

We will start by discussing the dependency of the dissipation and the non-Markovianity on the Rabi angle $\phi_{2}$ by $Z_{2}$ which addresses the state $|2\rangle$. We set $\phi_{2}=2 n \pi$ with $n$ being an integer in order to induce strong non-Markovianity and Zeno confinement as discussed in Sec. III A. In between, i.e., $\phi_{2} \neq 2 n \pi$, the degree of non-Markovianity vanishes quickly [compare inset in Fig. 2 (c)] which also allows access to the Markovian regime. In the following, we will show that $\phi_{2}$ tunes the non-Markovianity also in the presence of the second Zeno pulse $Z_{1}$.

Figure 4 shows the dissipation and the non-Markovianity as a function of the Rabi angle $\phi_{2}$ for $\phi_{1}=0.05 \pi$ and five different values of the displacement $\beta$. Panel (b) shows clearly that the non-Markovianity depends linearly on $\phi_{2}$. The dependency of the dissipation on $\phi_{2}$, on the other hand, is comparatively flat as can be seen in Fig. 4(a). However, the deviation from a constant value grows with $\beta$. Most notably does a Rabi angle with $\phi_{2}=(4 n+2) \pi$, and in particularly $2 \pi$, lead to more dissipation than $\phi_{2}=4 n \pi$. The reason for this lies in the 


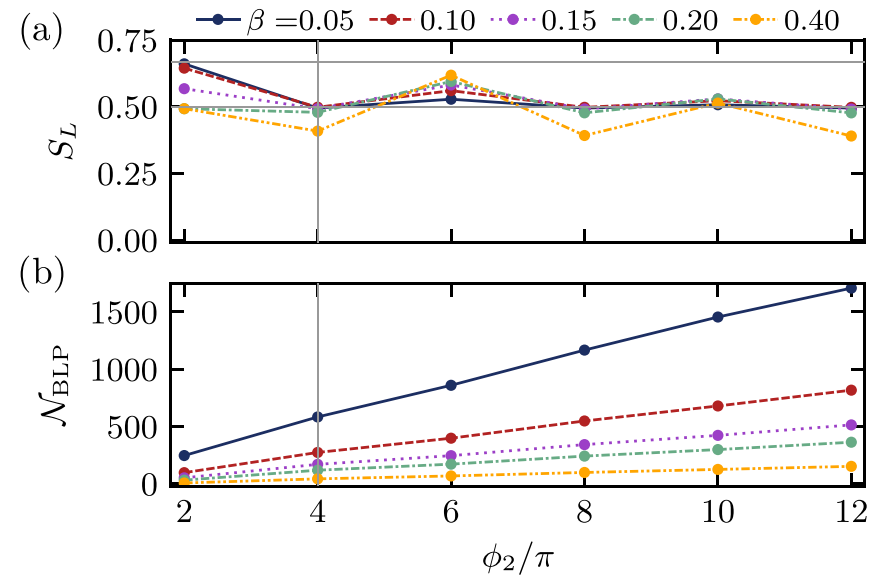

FIG. 4. Dependency of the dissipation $S_{L}$ (a) and the nonMarkovianity $\mathcal{N}_{\mathrm{BLP}}$ (b) on the Rabi angle $\phi_{2}$ for $\phi_{1}=0.05 \pi . \beta$ is fixed as given by the key. The horizontal gray lines in (a) indicate a linear entropy of $1 / 2$ and $2 / 3$, respectively. The vertical gray line indicates the value of $\phi_{1}$ shown in Fig. 5. The total duration $T$ of each propagation was set to fulfill $-i \alpha T=10 \pi$.

noncommutativity of the Hamiltonians $\hat{H}_{\mathrm{D}}$ [cf. Eq. (2)] and $\hat{H}_{z}$ [Eq. (3)]. For large interaction times $\tau$ (and accordingly large values of $\beta$ ), the pulse $Z_{z}$ is not able to perform a perfect $\phi_{z}$ pulse on the $|h, z\rangle \leftrightarrow|+, z\rangle$ transition because the dynamics is also affected by the drive $D$. As a consequence, the dressed state stays populated at the time of the measurement and dissipation occurs. A pulse with $\phi_{z}=4 n \pi$, however, is less affected by the noncommutativity of the operators because the first and second half of the Zeno pulse will differ in sign as shown in Eq. (6). In the same fashion as a spin-echo [42], the second half reverts the unwanted effects of the first one. As a result, a pulse with $\phi_{2}=4 n \pi$ will confine the dynamics of the system to the two-dimensional Zeno subspace created by it and the linear entropy cannot surpass the value of $1 / 2$.

We will now turn to discussing the dependency of the dissipation and the non-Markovianity on the Rabi angle $\phi_{1}$. We added the pulse $Z_{1}$ with the intention of inducing dissipation in the two-dimensional Zeno subspace of the system and, in the following, it will be shown that the dissipation $S_{L}$ depends particularly strong on $\phi_{1}$ if $\phi_{1}$ is very small.

As $\phi_{1}$ is switched on, the linear entropy rises rapidly from 0 to its maximal value $1 / 2$ while $\mathcal{N}_{\text {BLP }}$ varies sufficiently slow (cf. Fig. 5). This points to $\phi_{1}$ being a suitable knob to control the dissipation in the quantum simulator. Note that the maximal value of the non-Markovianity depends on $\beta$ since $\mathcal{N}_{\mathrm{BLP}}$ is proportional to the number $N$ of measurements performed during the total time $T$. Only for large values of $\beta$, e.g., $\beta=0.4$, the Zeno coupling induced by the pulse $Z_{2}$ is not sufficient to confine the dynamics to a two-dimensional Zeno subspace and the linear entropy takes values larger than $1 / 2$. In order to provide full tunability of the dissipation in the Zeno subspace of the system, it is sufficient to consider this range of $\phi_{1}$, where the dissipation takes all the possible values between 0 and $1 / 2$.

Nonetheless, we will also discuss the parameter range after the peak of $S_{L}$ in order to provide deep insight into the dynamics of the system. After the peak in dissipation and

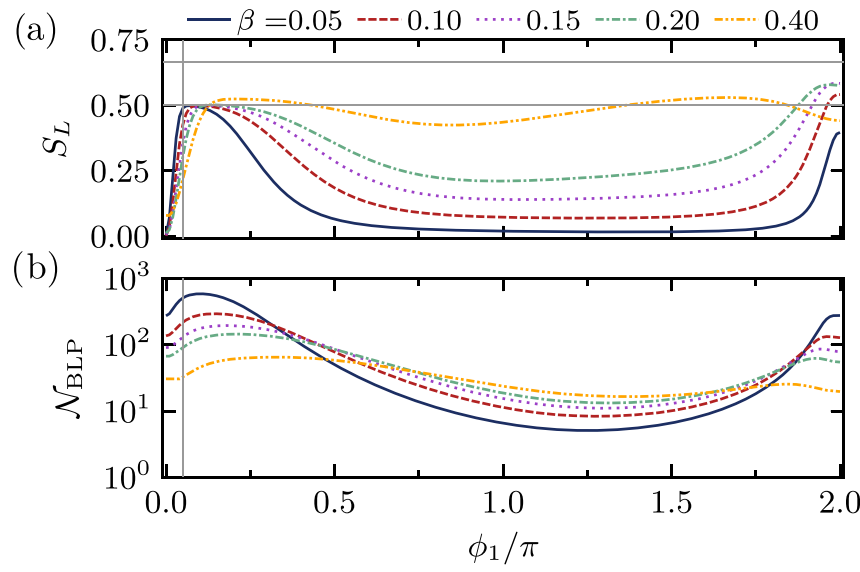

FIG. 5. Same as Fig. 4 but for variation of the Rabi angle $\phi_{1}$ with $\phi_{2}=4 \pi$. The vertical gray line indicates the value of $\phi_{1}$ shown in Fig. 4.

non-Markovianity, care must be taken since both quantities drop as $\phi_{1}$ is increased further. In this parameter regime, $Z_{1}$ induces Zeno confinement at $|1\rangle$ which prevents dissipation from occurring. The confinement is stronger the smaller $\beta$ and it also prevents a population of $|2\rangle$, which is the origin of memory effects, turning the dynamics Markovian. As $\phi_{1} \rightarrow$ $2 \pi$, the combination of two Zeno pulses $Z_{1}$ and $Z_{2}$ induce induce a strong disturbance of the initial state $|0\rangle$ while keeping it in the subspace $\{|0\rangle,|1\rangle,|2\rangle\}$. Hence, both the dissipation and the non-Markovianity rise significantly and the initial state will drift towards the completely mixed state with $S_{L}=2 / 3$ (compare to boundary in Fig. 3).

We want to stress that the Zeno pulses can not only be used to induce dissipation and non-Markovianity, but they can also confine the dynamics of the system to a subspace of adjustable size. In general, to confine the dynamics to a $d$-dimensional subspace, one simply needs to choose the Zeno level $|z=d\rangle$ by adjusting the frequency of the Zeno pulse $Z_{z}$ accordingly. The dimension of the system should therefore at least be three in order to enable nontrivial dynamics, but can be arbitrarily large and even infinite as in our setup. It is also possible to generate dissipation and memory effects on multiple system states $\left|z_{i}\right\rangle$ with $i \in\left\{1,2, \ldots, N_{Z}\right\}$ by varying the frequency of a single Zeno pulse $Z_{z}$ as a function of time or by employing multiple Zeno pulses $Z_{z_{i}}$. The number $N_{Z}$ of maximally controlled states is then only limited by the number of control pulses available.

\section{POSSIBLE EXPERIMENTAL IMPLEMENTATIONS}

The presented scheme can be realized experimentally in several ways. The proposed bipartite system of a harmonic oscillator and a three-level system is very natural and can be found in many experimental setups. One specific example is cavity QED, where the harmonic oscillator can be identified with one mode of microwave cavity and the three-level system with three neighboring circular states of a Rydberg atom, $|49 \mathrm{C}\rangle \equiv|h\rangle,|50 \mathrm{C}\rangle \equiv|g\rangle$, and $|51 \mathrm{C}\rangle \equiv|e\rangle$. In the following, we will discuss additional details on the realization of the presented scheme in a cavity QED setup such as as proposed in Ref. [35] by which our model was inspired. 
In this experiment, the Rydberg atoms interact with the microwave cavity in an atomic fountain arrangement: the atoms are injected into the cavity vertically from underneath while they are accelerate downwards by their own gravity. The turning point of the atom's trajectory is adjusted to be in the center of the cavity such that the interaction time between cavity and atom is sufficiently long to perform the experiment. The $|g\rangle \leftrightarrow|e\rangle$ transition of the Rydberg atom and the frequency $\omega$ of the cavity mode can be chosen to be resonant. The Rydberg atom then naturally fulfills the condition $\omega_{g e}=\omega \ll \omega^{\prime}=\omega_{h g}$, and transitions to higher or lower circular states are sufficiently far detuned from all other transitions. In the simulations presented above, we take the transition frequencies of the three-level system and the harmonic oscillator, and the Rabi frequency $\Omega$ from Ref. [35].

Due to the cryostatic environment of the experiment and the long lifetime of circular states in Rydberg atoms, "true" dissipation due to field energy damping or atomic relaxation is negligible on the relevant timescale. The atom will be excited to the Rydberg regime when it is already located inside the cavity which marks the beginning of one sequence of the protocol. In order to specifically address a $|h, z\rangle \leftrightarrow|+, z\rangle$ transition using a Zeno pulse $Z_{z}$ and distinguish it from the nearby $|h, z\rangle \leftrightarrow|-, z\rangle$ and $|h, z\rangle \leftrightarrow|-, z-1\rangle$ transitions, the duration $\Delta t_{Z}$ of the pulse $Z_{z}$ has to be long enough, namely $\Delta t_{Z} \gg 1 /(\Omega|\sqrt{z+1}-\sqrt{z}|)$ [35]. The end of one sequence with interaction time $\tau$ is triggered by ionizing the atom within the cavity by field ionization.

Of course, our scheme is not limited to cavity QED but an equivalent bipartite system can also be found in other experimental setups, such as a superconducting qubit coupled to a cavity in circuit QED [43], or a common vibrational mode of trapped ions together with ion qubit states [44]. Moreover, our approach is not restricted to modelling the system as a harmonic oscillator. In fact, not even a bipartite setup is required-it is sufficient to identify two different subspaces in the quantum system, the "system" and "meter" parts, and carry out state-selective measurements of the "system" using the "meter." An example for a realization in a single system would be a Bose-Einstein condensate (BEC) with hyperfine ground state levels encoding the system [45]. In general, realization of our proposal requires three conditions to be met: (i) existence of a system part whose dynamics are decoupled from the meter part when driven by a classical source $D$, (ii) selective excitation of system states by driving transitions in the meter using one or several Zeno pulses $Z_{z_{i}}$, (iii) subjecting meter states to dissipation. Dissipation can be introduced by projective measurement of the meter, as in the cavity QED example, or it can be natural such as a fast decay. In the BEC experiment, the Zeno pulse drives transitions to an excited state hyperfine manifold such that the meter is subject to spontaneous decay. Note that in this case, the number $N_{Z}$ of maximally controlled states is limited by the dimension of the meter subspace, i.e. the excited hyperfine manifold.

\section{CONCLUSIONS}

We have introduced a quantum simulator for openquantum-system dynamics with tunable non-Markovianity which is universal in the Markovian limit. It is based on a se- ries of indirect, state-selective measurements of a system by a meter. The open system dynamics can be tuned from memoryless to maximal display of memory effects by choosing the amount of entanglement between system and meter at the end of their interaction. Thus, our simulator directly realizes repeated interactions between system and environment and past-future (in)dependence [46]. The strength of dissipation and degree of non-Markovianity can be engineered independently by properly choosing the amplitudes of classical drive and Zeno pulses. The frequencies of the Zeno pulses, in turn, determine which system levels are subject to dissipation. Our proposal uses the quantum Zeno effect to open the path to experimentally study dissipation, memory effects, and their interplay in open quantum system dynamics in a controlled way. This will allow one to clarify the role of memory effects for the controllability of open quantum systems [47]. In more detail, we suggest to combine two experimental protocols-a specific control protocol (to prepare a desired state or implement a quantum gate) with the measurement-based open system simulator. Changing the type and strength of dissipation in the open system simulator will allow one to determine whether or not, and how well, the control protocol figure of merit can be optimized. In the same spirit, such a combination of quantum control and tunable dissipation opens the way to investigating how memory effects alter control strategies for open quantum systems as relevant for applications in quantum technology.

\section{ACKNOWLEDGMENTS}

We thank J.-M. Raimond, S. Gleyzes, F. Assémat, and E. M. Laine for discussions. Financial support from the European Union under the Research and Innovation action project "RYSQ"(Project No. 640378), the DAAD/Academy of Finland mobility grants, and the Studienstiftung des deutschen Volkes e.V. is gratefully acknowledged. S.M. acknowledges the Academy of Finland via the Projects No. 312058 and No. 287750 for financal support. This work was partly done using the high-performance computing cluster FUCHS-CSC provided by the Center for Scientific Computing (CSC) of the Goethe University Frankfurt in the framework of the HHLRGU (Hessisches Hochleistungsrechenzentrum der GoetheUniversität).

\section{APPENDIX A: CALCULATION OF THE NON-MARKOVIANITY MEASURE}

In order to quantify memory effects, we use the BLP measure [39]. It is defined as

$$
\mathcal{N}_{\mathrm{BLP}}=\max _{\rho_{1,2}(0)} \int_{\sigma>0} d t \sigma\left(\rho_{1,2}(0)\right),
$$

where $\sigma\left(\rho_{1,2}(0)\right)=\frac{d}{d t} D\left(\rho_{1}(t), \rho_{2}(t)\right)$ is the rate of change of the trace distance,

$$
D\left(\rho_{1}(t), \rho_{2}(t)\right)=\frac{1}{2} \operatorname{Tr}\left|\rho_{1}(t)-\rho_{2}(t)\right|,
$$

which is a measure of the distinguishability of two quantum states $\rho_{1}(t)$ and $\rho_{2}(t)$. The idea of the BLP measure is to integrate over all times where the distinguishability is increasing - a property which is characteristic for 


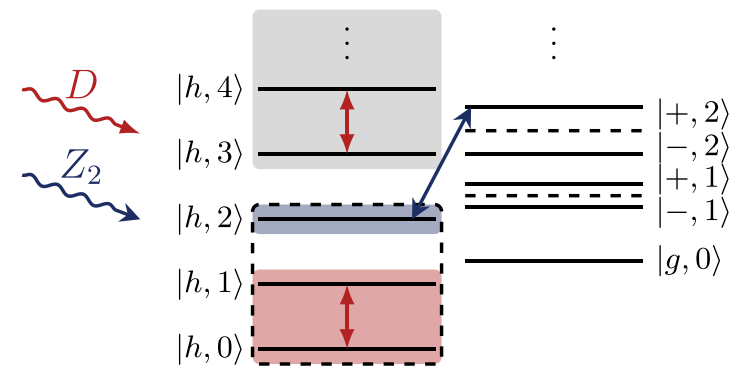

FIG. 6. Energy level diagram of the bipartite system indicating different subspaces: the Zeno subspace $\mathcal{H}_{Z}$ (red shaded), the Zeno level $|z\rangle$ (blue shaded), the extended Zeno subspace $\mathcal{H}_{Z}^{+}$(black dashed box), and the subspace with purely unitary dynamics (grey shaded).

non-Markovian dynamics. Evaluation of this measure requires comparatively little numerical effort-propagation of two well chosen initial states whereas the geometrical measure for non-Markovianity given by the state space volume [48], for instance, requires to propagate a full set of basis states. The measure proposed in [49] based on maximally entangling the system with an ancillary system leads to a further increase of the total dimension of the Hilbert space and is hence not practical for our purposes either. An obstacle for using the BLP measure is that it requires optimization over the system Hilbert space in Eq. (A1) in order to identify the initial state pair $\rho_{1,2}(0)$ which maximizes the measure-the so-called optimal state pair. On first glance, this seems difficult for the infinitely large Hilbert space of a harmonic oscillator. Nonetheless, the BLP measure turns out to be suitable in our case since we can reduce the size of the subspace in which we have to perform the optimization significantly by using our knowledge about the tailored interaction of the harmonic oscillator and the meter.

The reduction of the system size goes as follows, cf. Fig. 6 . We know that the Zeno pulse only affects the Zeno level and the level below since the dressed state we couple to is $|+, z\rangle=\frac{1}{\sqrt{2}}(|e, z-1\rangle+|g, z\rangle)$. If the initial state is located within the Zeno subspace $\mathcal{H}_{Z}$, signatures of non-Markovianity can only arise from population in states within the extended Zeno subspace $\mathcal{H}_{Z}^{+}=\{|0\rangle, \ldots,|z\rangle\}$, whereas the dynamics in the remaining Hilbert space are purely unitary. Numerical tests confirm this conjecture. It is thus sufficient to consider only the extended Zeno subspace for the optimization.

The optimization can be further simplified by considering properties of optimal state pairs for the BLP measure in general. From the definition in Eq. (A1) we can conclude that the trace distance of the optimal state pair shows revivals for the longest time with a maximal peak amplitude as compared to all other state pairs. Ideally, the states oscillate between being fully distinguishable, with trace distance equal to 1 , and fully indistinguishable, with trace distance equal to 0 . Thus, the dynamics of the two states which form the optimal pair should be as different from each other as possible. In a qubit, this can be realized by choosing the initial state pair to be orthogonal and on the boundary of the space of physical states [50]. In our model, the Zeno level and a state within the Zeno subspace form such a pair. The former is subject to the quantum Zeno effect which means that the population in the Zeno level is frozen. For the latter, quantum Zeno dynamics (QZD) are induced and the population never leaves the Zeno subspace. Thus, in an ideal Zeno situation, this pair of initial states stays distinguishable forever. However, in our realistic model with time-resolved dynamics, the Zeno pulse introduces Rabi oscillations in the bipartite system. Thus the overlap between the two states varies with time, leading to oscillations of the trace distance which indicate information flow and non-Markovianity. In the following, we seek to calculate the time-dependent trace distance of such a state pair explicitly.

We start by describing the dynamics of the first state of the pair: a state in the Zeno subspace. In the Zeno limit, every state in the Zeno subspace will be confined to it forever and will only be driven coherently by the drive $D$ [cf. Eq. (2)]. In the special case of $z=2$, an arbitrary state in the Zeno subspace can be parametrized by its Bloch angles,

$$
|\psi(\vartheta, \varphi)\rangle=\cos \left(\frac{\vartheta}{2}\right)|0\rangle+\sin \left(\frac{\vartheta}{2}\right) e^{i \varphi}|1\rangle,
$$

and the unitary evolution induced by $D$ takes the form

$$
\begin{aligned}
U_{z=2}(t) & =e^{-i\left(\alpha|1\rangle\left\langle 0\left|+\alpha^{*}\right| 0\right\rangle\langle 1|\right) t} \\
& =\cos (|\alpha| t)\left(\begin{array}{ll}
1 & 0 \\
0 & 1
\end{array}\right)-i \sin (|\alpha| t)\left(\begin{array}{cc}
0 & e^{-i \varphi_{\alpha}} \\
e^{i \varphi_{\alpha}} & 0
\end{array}\right)
\end{aligned}
$$

with $\alpha=|\alpha| e^{i \varphi_{\alpha}}$ being the amplitude of the field $D$. The eigenstates of this map are

$$
\left|\psi\left(\vartheta=\frac{\pi}{2}, \varphi= \pm \varphi_{\alpha}\right)\right\rangle=\frac{1}{\sqrt{2}}\left(|0\rangle+e^{ \pm i \varphi_{\alpha}}|1\rangle\right) .
$$

For our choice of $-i \alpha T=2 \pi$, we obtain $\varphi_{\alpha}=\pi / 2$ leading to $e^{ \pm i \varphi_{\alpha}}= \pm i$.

The second state of the pair is the Zeno level, $|h, z\rangle$, and in the Zeno limit the Zeno pulse will freeze the population in this state (quantum Zeno effect). On the timescale of the Zeno pulse, however, the pulse will induce oscillations between the initial state and the dressed state $|+, z\rangle$, as shown in Eq. (6). For $g_{z} t=\pi$, the final state is the dressed state. At this point, the reduced density matrix of the system will read

$$
\rho_{2}\left(t=\frac{\pi}{g_{z}}\right) \equiv \rho_{2}^{(\pi)}=\frac{1}{2}(|z-1\rangle\langle z-1|+| z\rangle\langle z|) .
$$

For $g_{z} t=2 \pi$, the population has oscillated back to the initial state.

We now consider the initial state pair $\left\{\left|\Psi_{1}\right\rangle=|\psi(\vartheta, \varphi)\rangle\right.$, $\left.\left|\Psi_{2}\right\rangle=|2\rangle\right\}$ for the special case of a Rabi angle $\phi_{2}=2 \pi$. In the beginning and end of each time interval $\tau$ the state $\left|\Psi_{2}\right\rangle$ can be found in the Zeno level, the overlap between the two states is zero and the trace distance is one. No information is destroyed during the measurement at this point because the system and the meter are separable. In between, $\left|\Psi_{2}(t)\right\rangle$ can be found in $\rho_{2}^{(\pi)}$ which has the largest overlap with the level $|z-1\rangle$ that is possible when starting in the Zeno level. The trace distance will thus oscillate quickly between 1 and the lower envelope given by the trace distance of $\left|\Psi_{1}(t)\right\rangle=$ $U_{z=2}(t)|\psi(\vartheta, \varphi)\rangle$ evolving in the Zeno subspace and $\left|\Psi_{2}(t)\right\rangle$ 


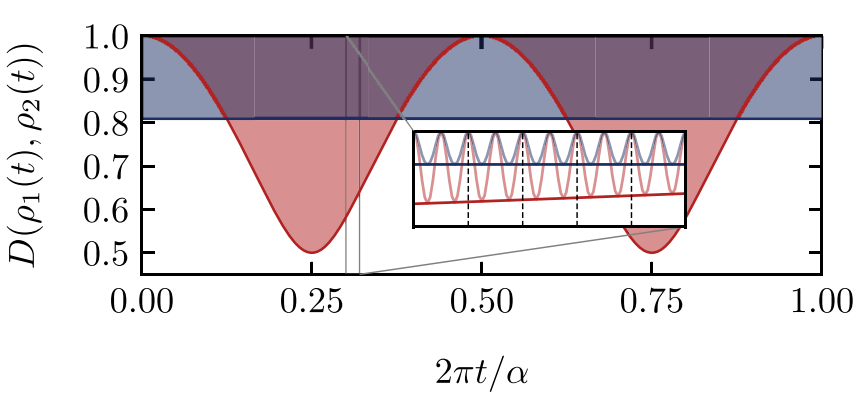

FIG. 7. Evolution of the trace distance for two different initial state pairs with Rabi angle $\phi_{2}=4 \pi$ and a displacement $\beta=$ 0.025. The state pairs are $\left|\Psi_{1}\right\rangle=|\psi(\vartheta=0, \varphi=0)\rangle=|0\rangle$ (red) and $\left|\Psi_{1}\right\rangle=\left|\psi\left(\vartheta=\frac{\pi}{2}, \varphi=\frac{\pi}{2}\right)\right\rangle=\frac{1}{\sqrt{2}}(|0\rangle+i|1\rangle)$ (blue) which is an eigenstate of $U_{z=2}(t)$, paired with the Zeno level $\left|\Psi_{2}\right\rangle=|2\rangle$. The dark lines show the result of the analytical calculation of the lower envelope, the light lines show the numerical calculations. The inset shows the fast oscillation of the trace distance due to the Zeno pulse $Z_{2}$ acting on $\left|\Psi_{2}\right\rangle$. The vertical black dashed lines show the positions of the meter's measurement.

being $\rho_{2}^{(\pi)}$. Note that if the Rabi angle is different from $\phi_{2}=$ $2 n \pi$, the system and meter will be entangled at the time of the measurement which decreases the trace distance with every measurement. As a result, the integrated trace distance and hence the BLP measure will be maximal for $\phi_{2}=2 n \pi$.

To calculate the lower envelope trace distance [cf. Eq. (A2)], we have to express both states in terms of density matrices. The density matrix of the state within the Zeno subspace is simply given by $\rho_{1}(t)=\left|\Psi_{1}(t)\right\rangle\left\langle\Psi_{1}(t)\right|$ while we assume the second state to be in mixed state $\rho_{2}^{(\pi)}$ [cf. Eq. (A6)] all the time. Afterwards, we have to calculate the eigenvalues of the matrix $\rho_{1-2}(t)=\rho_{1}(t)-\rho_{2}^{(\pi)}$. Using computer algebra and $\varphi_{\alpha}=\pi / 2$, we obtain

$$
\begin{aligned}
\lambda_{1}= & -\frac{1}{2} \\
\lambda_{2,3}= & \frac{1}{4} \pm \frac{1}{4} i e^{-i \varphi}\left\{e^{2 i \varphi}(4 \sin (\theta) \cos (\varphi) \sin (2|\alpha| t)\right. \\
& -4 \cos (\theta) \cos (2|\alpha| t)-5)\}^{1 / 2} .
\end{aligned}
$$

The envelope of the trace distance is then given by $D\left(\rho_{1}(t), \rho_{2}(t)\right)=\frac{1}{2} \sum_{i=1}^{3}\left|\lambda_{i}(t)\right|$. The result is shown for the two extremal cases in Fig. 7. If we choose $\left|\Psi_{1}\right\rangle$ to be one of the eigenstates of $U_{z=2}(t)$ [cf. Eq. (A5)], the envelope of the trace distance is constant (dark blue line). If we choose $\left|\Psi_{1}\right\rangle=|\psi(\vartheta, \varphi=\{0, \pi\})\rangle$ such that it lies on the meridian of the Bloch sphere (dark red) the envelope of the trace distance undergoes slow oscillations due to the drive $D$ [cf. Eq. (A4)]. The red curve in Fig. 7 shows the case $\left|\Psi_{1}\right\rangle=|0\rangle$ where $\vartheta=0$. A variation of $\vartheta$ will simply shift the curve on the time axis. This is because the drive $D$ will induce a rotation around the $y$-axis for our choice of $\varphi_{\alpha}=\pi / 2$ and every state that lies initially on that meridian will stay on it. In more general terms, the rotation axis $\vec{n}_{\alpha}$ depends on the phase of the complex amplitude $\alpha$ as

$$
\vec{n}_{\alpha}=\left(\cos \varphi_{\alpha}, \sin \varphi_{\alpha}, 0\right)=(\operatorname{Re} \alpha, \operatorname{Im} \alpha, 0) /|\alpha| .
$$

The eigenstates are given by Eq. (A5) and the states with $\varphi= \pm \varphi_{\alpha}+\pi / 2$ will evolve on a meridian through the Bloch sphere's poles.

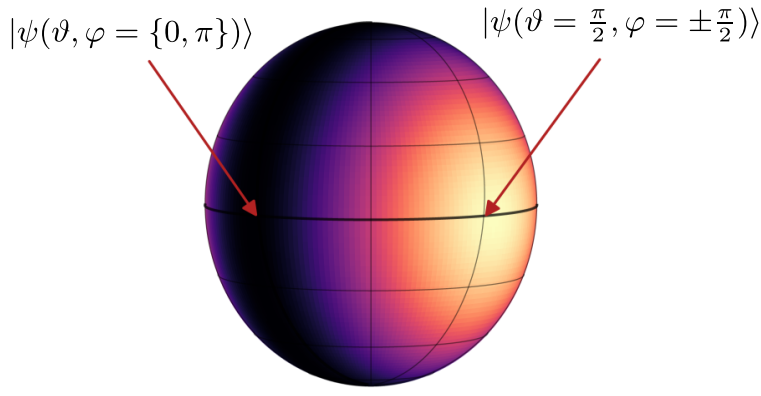

$\max$.

$\mathcal{N}_{\mathrm{BLP}}$

$\min$

FIG. 8. BLP measure $\mathcal{N}_{\text {BLP }}$ for different initial states $\left|\Psi_{1}\right\rangle=$ $|\psi(\vartheta, \varphi)\rangle$ on the Bloch sphere spanned by the Zeno subspace $\{|0\rangle,|1\rangle\}$. The second state of the state pair is the Zeno level $\left|\Psi_{2}\right\rangle=$ |2) outside of this subspace. The parameters are $\beta=0.025$ and $\phi_{2}=$ $4 \pi$. The data was obtained numerically but the analytical expression in Eq. (A7) gives the same results.

The value of the BLP measure is now connected to the area enclosed by the lower envelope and the $D=1$ line in Fig. 7 . The trace distance oscillates between the lower envelope and 1 every time a $2 \pi$ pulse is completed on the $|h, 2\rangle \leftrightarrow|+, 2\rangle$ transition (see light lines indicating the numerical calculations). Since the BLP measure sums up all the areas where the trace distance is increasing [cf. Eq. (A1)], we have to sum $n$ peaks per time interval $\tau$ for $\phi_{2}=2 n \pi$ (see inset in Fig. 7, there are $n=\phi_{2} / 2 \pi=2$ peaks between two measurements). This leads to the final expression

$$
\mathcal{N}_{\mathrm{BLP}}=\sum_{j=1}^{n N}\left(1-D\left(\frac{j \tau}{n}\right)\right)
$$

where $T=N \tau$. Note the remarkable agreement of the analytical envelope and the numerical calculations in Fig. 7 albeit the assumption of a perfect Zeno confinement. Finally, Fig. 8 shows the BLP measure for different initial states $\left|\Psi_{1}\right\rangle$. The BLP measure takes a minimum for the eigenstates of $U_{z=2}(t)$ and a maximum for the states on the meridian with $\varphi=\{0, \pi\}$. This is because the blue area in Fig. 7 which describes the evolution of the trace distance for the eigenstates is smaller than the red are which describes the states on the meridian.

Summing up, the optimal state pair which leads to the maximal value of the BLP measure was found to be composed of the state $\left|\Psi_{1}\right\rangle=\left|\psi\left(\vartheta, \varphi= \pm \varphi_{\alpha}+\pi / 2\right)\right\rangle$ which lives in the Zeno subspace spanned by $\{|0\rangle,|1\rangle\}$ and depends on the phase $\varphi_{\alpha}$ of the complex amplitude $\alpha$ of the drive $D$, and the Zeno level $|z=2\rangle$. Note that the calculations can be generalized to larger values of $z>2$. The larger dimension of $U_{z}(t)$ [cf. Eq. (A4)] will turn the equations more complicated but the central results remain unchanged.

When considering two Zeno pulses, $Z_{1}$ and $Z_{2}$, to induce non-Markovianity and dissipation, the optimal state pair is possibly different since all three states in the extended Zeno subspace $\mathcal{H}_{Z}^{+}$take part in the nonunitary 
dynamics induced by the Zeno coupling and the measurement. A numerical reoptimization was performed for the specific case of $\left\{\beta=0.025, \phi_{2}=4 \pi, \phi_{1}=0.25 \pi\right\}$ providing both dissipation $\left(S_{L}=0.48\right)$ and a large amount of nonMarkovianity (about twice as large as without having the second Zeno pulse $\left.Z_{1}\right)$. After optimizing $\left|\Psi_{1}\right\rangle$ numerically, the optimal state pair for this set of parameters was found to be $\left\{\left|\Psi_{1}\right\rangle=|\psi(\vartheta=0.56 \pi, \varphi=1.92 \pi)\rangle,\left|\Psi_{2}\right\rangle=|2\rangle\right\}$. To be exact, this optimization would have to be performed for all considered parameters $\left\{\beta, \phi_{2}, \phi_{1}\right\}$ and with considering choices other than $\left|\Psi_{1}\right\rangle=|\psi(\vartheta, \varphi)\rangle$ and $\left|\Psi_{2}\right\rangle=|2\rangle$ for the optimal state pair. However, the minimal and maximal value of the BLP measure differ by about $1 \%$ only and since the absolute value of the BLP measure is not important, we choose $\left\{\left|\Psi_{1}\right\rangle=\frac{1}{\sqrt{2}}(|0\rangle+|1\rangle),\left|\Psi_{2}\right\rangle=|2\rangle\right\}$ throughout for simplicity.

\section{APPENDIX B: DERIVATION OF THE LINDBLAD MASTER EQUATION}

The most general way to describe the dynamics of a Markovian open quantum system is by means of a Gorini-Kossakowski-Sudarshan-Lindblad equation. The explicit form of the decay rates and Lindblad operators gives information on the kind of dissipation occurring in an open quantum system and it provides us with a recipe on how to control the open-quantum-system simulator. In the following, we show the detailed derivation of the master equation in Lindblad form shown in Eq. (7).

We start from the piecewise dynamics of Eq. (4) which describes the reduced state of the system after interacting with a meter during time $\tau$. When writing down this equation, we have assumed to employ a single Zeno pulse $Z_{z}$ which acts on an arbitrary Zeno level $|z\rangle$. The first step is to evaluate the partial trace and to derive the Krauss representation of the dynamical map. To this end, we expand the time evolution operator $\hat{U}(d t)$ using the Baker-Campbell-Hausdorff formula up to first order,

$$
\hat{U}(d t)=e^{-i\left(\hat{H}_{\mathrm{D}}+\hat{H}_{\mathrm{sm}}+\hat{H}_{\mathrm{z}}\right) d t} \approx e^{-i \hat{H}_{\mathrm{D}} d t} e^{-i \hat{H}_{\mathrm{sm}} d t} e^{-i \hat{H}_{\mathrm{z}} d t} .
$$

The expression for the unitary evolution of the bipartite state, $\hat{U}(\tau) \hat{\rho}(t) \hat{U}^{\dagger}(\tau)$, can be simplified using two properties. First, we start each sequence of coupling the system to a meter with the separable initial state $\hat{\rho}(t)=\hat{\rho}_{\mathrm{s}}(t) \otimes|h\rangle\langle h|$, where $\hat{\rho}_{\mathrm{s}}(t)$ is the reduced state of the system and $|h\rangle$ is the initial state of the meter. Second, it is easy to see that $\left[\hat{H}_{\mathrm{sm}}, \hat{\rho}_{\mathrm{s}}(t) \otimes|h\rangle\langle h|\right]=0$ since $\hat{H}_{\mathrm{sm}}$ [cf. Eq. (1)] couples the system to the $|g\rangle \leftrightarrow|e\rangle$ transition of the meter. By combining both, we can evaluate the partial trace explicitly and we arrive at

$$
\hat{\rho}_{\mathrm{s}}(t+d t)=\sum_{j=h, g, e} w_{j h} \hat{U}_{\mathrm{D}}(d t) \hat{\rho}_{\mathrm{s}}(t) \hat{U}_{\mathrm{D}}^{\dagger}(d t) w_{j h}^{\dagger}
$$

with $\quad \hat{U}_{\mathrm{D}}(d t)=e^{-i \hat{H}_{\mathrm{D}} d t} \quad$ and $\quad w_{j h}=\left\langle j\left|\hat{U}_{\mathrm{z}}(d t)\right| h\right\rangle \quad$ with $\hat{U}_{\mathrm{Z}}(d t)=e^{-i \hat{H}_{z} d t}$. To describe the dynamics in its most general form, we write the Zeno Hamiltonian as

$$
\hat{H}_{z}=\frac{g_{(z, n)}}{2}(|h, z\rangle\langle+, n|+|+, n\rangle\langle h, z|)
$$

with $n$ and $z$ being arbitrary. Next, the unitary $\hat{U}_{\mathrm{z}}(d t)$ can be rewritten as

$$
\begin{aligned}
\hat{U}_{z}(d t)= & \hat{\mathbb{1}}-\left(1-\cos \frac{\phi_{(z, n)}}{2}\right)(|h, z\rangle\langle h, z|+|+, n\rangle\langle+, n|) \\
& -i \sin \frac{\phi_{(z, n)}}{2}(|h, z\rangle\langle+, n|+|+, n\rangle\langle h, z|)
\end{aligned}
$$

with the Rabi angle $\phi_{(z, n)}=g_{(z, n)} d t$. Using this, the operators $w_{j h}$ can be calculated to

$$
\begin{aligned}
& w_{h h}=\hat{\mathbb{1}}_{\mathrm{s}}-\left(1-\cos \frac{\phi_{(z, n)}}{2}\right) \hat{\Pi}_{z}, \\
& w_{g h}=-\frac{i}{\sqrt{2}} \sin \frac{\phi_{(z, n)}}{2} \hat{L}_{(z, n)}, \\
& w_{e h}=-\frac{i}{\sqrt{2}} \sin \frac{\phi_{(z, n)}}{2} \hat{L}_{(z, n-1)},
\end{aligned}
$$

where

$$
\hat{\Pi}_{z}=|z\rangle\left\langle z\left|, \quad \hat{L}_{(z, m)}=\right| m\right\rangle\langle z|
$$

are system operators. Note that Eq. (B1) can be interpreted both as the description of a measurement process with three measurement operators and as the Krauss representation of an open quantum system's evolution. The operators depend only on the Zeno Hamiltonian of Eq. (B2) and the meter's initial state and therefore the generalisation of the derivation to systems other than the harmonic oscillator is straightforward.

Furthermore, a time-continuous master equation in Lindblad form can be derived by assuming the measurement to be performed continuously with constant rate $\kappa=1 / \tau$ such that the number of measurements in a time interval $d t$ is $\kappa d t$. This can be interpreted as the "smearing" of one measurement over the whole time interval $\tau$. As a result, the master equation will not describe the same evolution as the piecewise dynamics for large values of $\tau$. Using this assumption and going to the interaction picture with respect to the coherent evolution $\hat{H}_{\mathrm{D}}$, we can rewrite Eq. (B1) to [51,52]

$$
\hat{\rho}_{\mathrm{s}}(t+d t)=\kappa d t \sum_{j=h, g, e} w_{j h} \hat{\rho}_{\mathrm{s}}(t) w_{j h}^{\dagger}+(1-\kappa d t) \hat{\rho}_{\mathrm{s}}(t) .
$$

Finally, we calculate the derivative of the reduced state as $\frac{d \hat{\rho}_{\mathrm{s}}(t)}{d t}=\lim _{d t \rightarrow 0} \frac{\hat{\rho}_{\mathrm{s}}(t+d t)-\hat{\rho}_{\mathrm{s}}(t)}{d t}$ and go back to the noninteracting picture [51,52],

$$
\frac{d \hat{\rho}_{\mathrm{s}}(t)}{d t}=-i\left[\hat{H}_{\mathrm{D}}, \hat{\rho}_{\mathrm{s}}\right]+\kappa\left(\sum_{j=h, g, e} w_{j h} \hat{\rho}_{\mathrm{s}}(t) w_{j h}^{\dagger}-\hat{\rho}_{\mathrm{s}}(t)\right) .
$$

The Krauss operators of Eq. (B3) can be inserted and the terms rearranged using that $\hat{\Pi}_{z}$ is idempotent and $\hat{\Pi}_{z}=\hat{L}_{(z, m)}^{\dagger} \hat{L}_{(z, m)}$. This leads to the Lindblad operators $\hat{L}_{(z, n)}, \hat{L}_{(z, n-1)}$ and $\hat{\Pi}_{z}$ with the decay rates

$$
\begin{aligned}
\gamma_{(z, n)} & =\gamma_{(z, n-1)}=\frac{1}{2} \sin ^{2}\left(\frac{\phi_{(z, n)}}{2}\right), \\
\gamma_{z} & =4 \sin ^{4}\left(\frac{\phi_{(z, n)}}{4}\right) .
\end{aligned}
$$




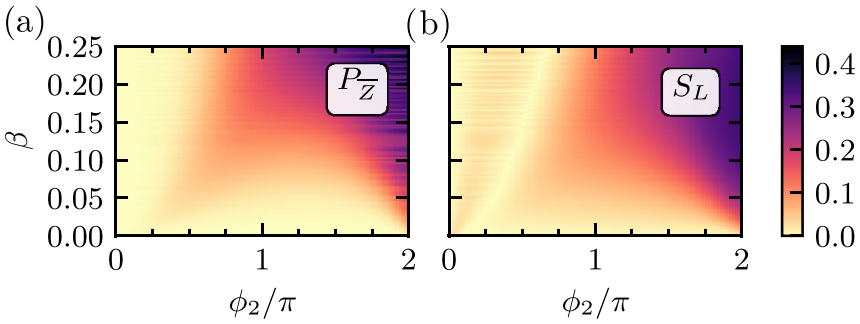

FIG. 9. Difference obtained when solving the master equation and using the piecewise dynamics for the Zeno infidelity $P_{\bar{Z}}$ (a) and the dissipation $S_{L}$ (b) as a function of the displacement $\beta$ due to the drive $D$ and the Rabi angle $\phi_{2}$ accumulated due to the Zeno pulse $Z_{2}$.

Finally, when choosing $n=z$ and with $\hat{\Pi} \equiv \hat{\Pi}_{z}=\hat{L}_{(z, z)}=$ $|z\rangle\left\langle z\left|, \hat{A} \equiv \hat{L}_{(z, z-1)}=\right| z-1\right\rangle\langle z|, \gamma_{\Pi} \equiv \gamma_{z}+\gamma_{(z, z)}$ and $\gamma_{A} \equiv$ $\gamma_{(z, z-1)}$ we arrive at the master equation as shown in Eq. (7).

To assess the agreement of the master equation (7) with the piecewise dynamics of Eq. (4), we propagate the initial state $|0\rangle$ with $z=2$ using both methods. Figure 9 shows the difference in infidelity of the QZD, $P_{\bar{Z}}$, and in the linear entropy $S_{L}$ between the two pictures. It can be seen that they show good agreement for small Rabi angles $\phi_{2}$ or displacements $\beta$. However, the two pictures deviate if our assumptions during the derivation of the master equation are violated. Firstly, the agreement deteriorates for large $\beta$, since this contradicts the assumption of continuous measurements, $d t \rightarrow 0$. Secondly, we have used the Baker-Campbell-Hausdorff formula up to first order to derive the Krauss representation. We thus make an error which is of the order of the commutator of $\hat{H}_{\mathrm{D}}$ and $\hat{H}_{\mathrm{z}}$ which scales as $\phi_{z} \beta$. Therefore the deviation also rises as $\phi_{2}$ increases.

Next, we want to discuss the master equation for some other cases. For $n=z+1$, we arrive with the Lindblad operators

$$
\hat{B} \equiv \hat{L}_{(z, z+1)}=|z+1\rangle\left\langle z\left|, \quad \hat{\Pi}=\hat{\Pi}_{z}=\hat{L}_{(z, z)}=\right| z\right\rangle\langle z|
$$

with the decay rates $\gamma_{\Pi}=\gamma_{z}+\gamma_{(z, z)}$ and $\gamma_{B} \equiv \gamma_{(z, z+1)}$. This case is similar to the one above with $n=z$, but the Lindblad operator $\hat{B}$ now describes population excitation instead of relaxation. Going to the off-resonant case allows for even broader generalization where off-resonant refers to the transition frequencies of the harmonic oscillator $\omega_{s}$ and the $|g\rangle \leftrightarrow|e\rangle$ transition of the meter $\omega_{e g}$ with $\Delta=\omega_{e g}-\omega_{s}$. For large detuning $\Delta \gg \Omega$, the dressed states tend towards the uncoupled ones, $|+, n\rangle \sim|e, n-1\rangle$ and $|-, n\rangle \sim|g, n\rangle$ [36]. The remaining interaction leads to a slight shift of the bipartite energy levels which still allows for state selective excitation. If we now couple the $|h, z\rangle \leftrightarrow|e, n\rangle$ transition with a Zeno pulse, we are able to create the Lindblad operators $\hat{L}_{(z, n)}=$ $|n\rangle\langle z|$ and $\hat{\Pi}_{z}=|z\rangle\langle z|$ where the corresponding decay rate relate to the resonant case in Eq. (B4) as $\gamma_{(z, n)}^{e} \equiv 2 \gamma_{(z, n)}$ and $\gamma_{z}^{e} \equiv \gamma_{z}$, respectively (the superscript $e$ refers to the coupling to the meter state $|e, n\rangle)$. The ratio between the two decay rates is given by

$$
\frac{\gamma_{(z, n)}^{e}}{\gamma_{z}^{e}}=\cot ^{2}\left(\frac{\phi_{(z, n)}}{4}\right)
$$

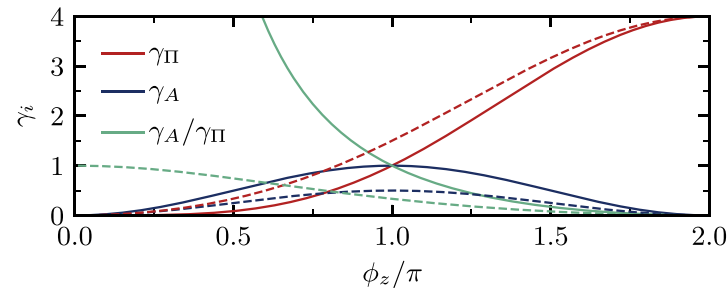

FIG. 10. Decay rates, $\gamma_{A}$ and $\gamma_{\Pi}$, and their ratio for the offresonant case coupling $|h, z\rangle \leftrightarrow|e, n=z-1\rangle$ (solid lines) and the resonant case coupling $|h, z\rangle \leftrightarrow|+, n=z\rangle$ (dashed lines).

Figure 10 shows the decay rates in the resonant case with $n=z$ (dashed lines) and the off-resonant case with $n=z-1$ (solid line) which both induce the same Lindblad operators $\hat{\Pi}=|z\rangle\langle z|$ and $\hat{A}=|z-1\rangle\langle z|$. In the resonant case, the ratio never exceeds the value 1 rendering it impossible to engineer decay rates with $\gamma_{A}>\gamma_{\pi}$. In the off-resonant case, on the other hand, the ratio varies between $\infty$ for $\phi_{z}=0$ and 0 for $\phi_{z}=2 \pi$. By additionally adjusting the measurement rate $\kappa$, by which all the decay rates have to be multiplied, it is possible to tune the ratio and the amplitude of the decay rates arbitrarily. Finally, in the special case of $n=$ $z$ in the off-resonant case, the pulse induces pure dephasing $\hat{L}_{(z, z)}=\hat{\Pi}_{z}=|z\rangle\langle z|$ with the decay rate $\gamma_{z}^{e}=\gamma_{z}+2 \gamma_{(z, z)}$ [cf. Eq. (B4)]. In the same spirit, we can couple $|h, z\rangle$ to $|g, n\rangle$ in the off-resonant case. This case is completely analogous to coupling to $|e, n\rangle$ but gives yet another degree of freedom to tailor the open systems dynamics.

While one might think that generating arbitrary sets of Lindblad operators is hampered by connections between them due to the Rabi angles, the number of linked decay rates is actually very small. For instance, a coupling of the Zeno level $|h, z\rangle$ to the dressed state $|+, n\rangle$ will induce the three Lindblad operators $\hat{\Pi}_{z}=|z\rangle\left\langle z\left|, \hat{L}_{(z, n)}=\right| n\right\rangle\langle z|$ and $\hat{L}_{(z, n-1)}=|n-1\rangle\langle z|$ whose decay rates $\gamma_{z}, \gamma_{(z, n)}$ and $\gamma_{(z, n-1)}$, cf. Eq. (B4), all depend on the same Rabi angle $\phi_{(z, n)}$. This can be reduced to two Lindblad operators, $\hat{\Pi}_{z}=|z\rangle\langle z|$ and $\hat{L}_{(z, n)}=|n\rangle\langle z|$, or even a single one describing pure dephasing $\hat{\Pi}_{z}$ (for $n=z$ ) simply by going to the off-resonant case, as explained above.

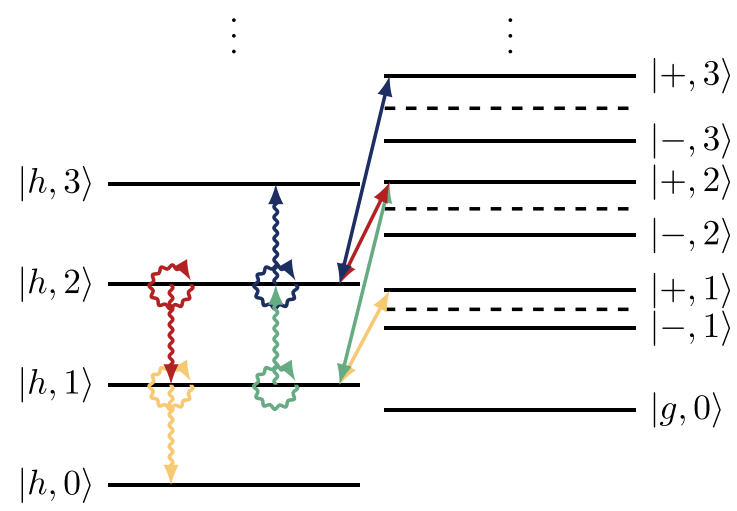

FIG. 11. Decay channels in the resonant case. The straight arrows in the middle of each panel indicate the driven transitions, the snaked arrows indicate the effective decay channels in the master equation. 
In other words, only between one to three decay rates depend on the same Rabi angle.

The key to engineering decay rates which are independent from each other is to combine several Zeno pulses $Z_{z_{i}, n_{i}}$ which address different transitions $\left|h, z_{i}\right\rangle \leftrightarrow\left|+, n_{i}\right\rangle$. The Rabi angles $\phi_{\left(z_{i}, n_{i}\right)}$ can then be tuned for each pulse independently leading to independent decay rates. Figure 11 shows the combination of four exemplary Zeno pulses in the resonant case. The only exception to the compatibility of the pulses is that the same dressed state $|+, m\rangle$ cannot be addressed with several pulses without introducing correlations, possibly undesired ones, of the decay channels. For instance, we cannot address the $|h, 2\rangle \rightarrow|+, 2\rangle$ transition (red arrow), inducing dephasing on $|2\rangle$ and deexcitation $|2\rangle \rightarrow|1\rangle$, and the $|h, 1\rangle \rightarrow|+, 2\rangle$ transition (green arrow), inducing dephasing on $|1\rangle$ and excitation $|1\rangle \rightarrow|2\rangle$, at the same time because both address $|+, 2\rangle$. This does not, however, reduce the generality of the scheme since simultaneous deexcitation, $|2\rangle \rightarrow|1\rangle$, and excitation, $|1\rangle \rightarrow|2\rangle$, can be replaced by an effective (de)excitation rate.
[1] I. M. Georgescu, S. Ashhab, and F. Nori, Rev. Mod. Phys. 86, 153 (2014)

[2] S. Maniscalco, J. Piilo, F. Petruccione, and A. Messina, Acta Physica Hungarica Series B, Quantum Electronics 23, 67 (2005).

[3] J. T. Barreiro, M. Müller, P. Schindler, D. Nigg, T. Monz, M. Chwalla, M. Hennrich, C. F. Roos, P. Zoller, and R. Blatt, Nature (London) 470, 486 (2011).

[4] B.-H. Liu, L. Li, Y.-F. Huang, C.-F. Li, G.-C. Guo, E.-M. Laine, H.-P. Breuer, and J. Piilo, Nat. Phys. 7, 931 (2011).

[5] S. Mostame, P. Rebentrost, A. Eisfeld, A. J. Kerman, D. I. Tsomokos, and A. Aspuru-Guzik, New J. Phys. 14, 105013 (2012).

[6] A. Chiuri, C. Greganti, L. Mazzola, M. Paternostro, and P. Mataloni, Sci. Rep. 2, 968 (2012).

[7] R. Sweke, I. Sinayskiy, and F. Petruccione, Phys. Rev. A 90, 022331 (2014).

[8] R. Sweke, I. Sinayskiy, D. Bernard, and F. Petruccione, Phys. Rev. A 91, 062308 (2015).

[9] R. Sweke, M. Sanz, I. Sinayskiy, F. Petruccione, and E. Solano, Phys. Rev. A 94, 022317 (2016).

[10] N. K. Bernardes, J. P. S. Peterson, R. S. Sarthour, A. M. Souza, C. H. Monken, I. Roditi, I. S. Oliveira, and M. F. Santos, Sci. Rep. 6, 33945 (2016).

[11] U. Alvarez-Rodriguez, R. Di Candia, J. Casanova, M. Sanz, and E. Solano, Phys. Rev. A 95, 020301(R) (2017).

[12] A. Chenu, M. Beau, J. Cao, and A. del Campo, Phys. Rev. Lett. 118, 140403 (2017)

[13] L. C. G. Govia, B. G. Taketani, P. K. Schuhmacher, and F. K. Wilhelm, Quantum Sci. Technol. 2, 015002 (2017).

[14] E. Mascarenhas and I. de Vega, Phys. Rev. A 96, 062117 (2017).

[15] H.-B. Chen, C. Gneiting, P.-Y. Lo, Y.-N. Chen, and F. Nori, Phys. Rev. Lett. 120, 030403 (2018).

[16] Z.-D. Liu, H. Lyyra, Y.-N. Sun, B.-H. Liu, C.-F. Li, G.-C. Guo, S. Maniscalco, and J. Piilo, Nat. Commun. 9, 3453 (2018).

[17] R. Uzdin, S. Gasparinetti, R. Ozeri, and R. Kosloff, New J. Phys. 20, 063030 (2018).

[18] A. Cuevas, A. Geraldi, C. Liorni, L. D. Bonavena, A. D. Pasquale, F. Sciarrino, V. Giovannetti, and P. Mataloni, Sci. Rep. 9, 3205 (2019).

[19] G. García-Pérez, M. A. C. Rossi, and S. Maniscalco, npj Quantum Inform. 6, 1 (2020).

[20] Á. Rivas, S. F. Huelga, and M. B. Plenio, Rep. Prog. Phys. 77, 094001 (2014).
[21] H.-P. Breuer, E.-M. Laine, J. Piilo, and B. Vacchini, Rev. Mod. Phys. 88, 021002 (2016).

[22] C. F. Li, G. C. Guo, and J. Piilo, Europhys. Lett. 127, 50001 (2019).

[23] U. Weiss, Quantum Dissipative Systems, 4th ed. (World Scientific, 2012).

[24] I. de Vega and D. Alonso, Rev. Mod. Phys. 89, 015001 (2017).

[25] B. Bhattacharya and S. Bhattacharya, arXiv:1805.11418.

[26] S. Bhattacharya, B. Bhattacharya, and A. S. Majumdar, arXiv: 1803.06881

[27] K.-D. Wu, Z. Hou, G.-Y. Xiang, C.-F. Li, G.-C. Guo, D. Dong, and F. Nori, arXiv:1903.03359.

[28] N. Anand and T. A. Brun, arXiv:1903.03880.

[29] C. Li, G. Guo, and J. Piilo, Europhys. Lett. 128, 30001 (2019).

[30] R. Schmidt, A. Negretti, J. Ankerhold, T. Calarco, and J. T. Stockburger, Phys. Rev. Lett. 107, 130404 (2011).

[31] E.-M. Laine, H.-P. Breuer, and J. Piilo, Sci. Rep. 4, 4620 (2014).

[32] B. Bylicka, D. Chruściński, and S. Maniscalco, Sci. Rep. 4, 5720 (2014).

[33] P. Rebentrost, I. Serban, T. Schulte-Herbrüggen, and F. K. Wilhelm, Phys. Rev. Lett. 102, 090401 (2009).

[34] D. M. Reich, N. Katz, and C. P. Koch, Sci. Rep. 5, 12430 (2015).

[35] J. M. Raimond, P. Facchi, B. Peaudecerf, S. Pascazio, C. Sayrin, I. Dotsenko, S. Gleyzes, M. Brune, and S. Haroche, Phys. Rev. A 86, 032120 (2012).

[36] S. Haroche and J.-M. Raimond, Exploring the Quantum. Atoms, Cavities, and Photons (Oxford University Press, New York, 2006).

[37] P. Facchi and S. Pascazio, Phys. Rev. Lett. 89, 080401 (2002).

[38] P. Facchi and S. Pascazio, J. Phys. A 41, 493001 (2008).

[39] H.-P. Breuer, E.-M. Laine, and J. Piilo, Phys. Rev. Lett. 103, 210401 (2009).

[40] P. Facchi and S. Pascazio, Proceedings 12, 30 (2019).

[41] G. Nogues, A. Rauschenbeutel, S. Osnaghi, M. Brune, J.-M. Raimond, and S. Haroche, Nature 400, 239 (1999).

[42] E. L. Hahn, Phys. Rev. 80, 580 (1950).

[43] A. Blais, R. S. Huang, A. Wallraff, S. M. Girvin, and R. J. Schoelkopf, Phys. Rev. A 69, 062320 (2004).

[44] D. Kienzler, H.-Y. Lo, B. Keitch, L. de Clercq, F. Leupold, F. Lindenfelser, M. Marinelli, V. Negnevitsky, and J. P. Home, Science 347, 53 (2015).

[45] F. Schäfer, I. Herrera, S. Cherukattil, C. Lovecchio, F. S. Cataliotti, F. Caruso, and A. Smerzi, Nat. Commun. 5, 3194 (2014). 
[46] L. Li, M. J. Hall, and H. M. Wiseman, Phys. Rep. 759, 1 (2018).

[47] C. P. Koch, J. Phys.: Condens. Matter 28, 213001 (2016).

[48] S. Lorenzo, F. Plastina, and M. Paternostro, Phys. Rev. A 88, 020102 (2013).

[49] Á. Rivas, S. F. Huelga, and M. B. Plenio, Phys. Rev. Lett. 105, 050403 (2010).
[50] S. Wißmann, A. Karlsson, E.-M. Laine, J. Piilo, and H.-P. Breuer, Phys. Rev. A 86, 062108 (2012).

[51] F. Lucas and K. Hornberger, Phys. Rev. Lett. 110, 240401 (2013).

[52] F. Lucas and K. Hornberger, Phys. Rev. A 89, 012112 (2014). 